\title{
Two remarks on the Weierstrass flag
}

\author{
Enrico Arbarello and Gabriele Mondello
}

\begin{abstract}
We show that the locally closed strata of the Weierstrass flags on $M_{g}$ and $M_{g, 1}$ are almost never affine.
\end{abstract}

\section{Introduction}

The coarse moduli space $M_{g, n}$ of curves of genus $g$ with $n$ marked points is a quasi-projective variety. Grothendieck 9] already wondered how many affines are needed to cover $M_{g, n}$. A first hint came from Diaz's upper bound [5] on the dimension of a complete subvariety of $M_{g, n}$, then strengthened by Harer's computation [10 of the virtual cohomological dimension of the mapping class group. Looijenga's vanishing 11] of the tautological classes in high degree motivated the following.

Question (Looijenga): Does $M_{g}$ have an affine stratification with $g-1$ layers? Does $M_{g, 1}$ have an affine stratification with $g$ layers?

In both cases, stratifications with the right number of layers do exist and it is natural to ask whether the layers are affine.

In this short paper, we will concentrate on the Weierstrass flag studied by Arbarello [2, whose strata had already been defined by Rauch [13. As Mumford pointed out in [12, Section 7, the proof of Theorem (3.27) in $[2$ is incomplete. Therefore, it is still not known whether one may exclude that any single stratum of this flag contains compact curves. Actually, one may even ask whether these strata are affine.

In this note, we will show that almost no such stratum is affine.

Clearly, these negative results about a specific stratification do not conflict with Looijenga's question. In fact, for $g \leq 5$, Fontanari-Looijenga [7] show that a good stratification exists and Fontanari-Pascolutti $[\mathbf{8}$ exhibit a good affine cover of $M_{g}$.

1.1. Content of the paper. Proposition 3.1 deals with the Weierstrass stratification in $M_{g, 1}$. The idea is to show that the strata can be realized as open subsets of smooth varieties, whose complement (representing a certain family of plane curves) is not purely divisorial. The techniques are borrowed from Arbarello-Cornalba [3] (see also Chapter XXI of [1]). Similar computations can be also found in CaporasoHarris [4.

Proposition 3.2 deals with the stratification of $M_{g}$ and relies on the same idea. The key computation is borrowed from Diaz [6].

We work over the field of complex numbers, but all the results hold over an algebraically closed field of characteristic zero.

2010 Mathematics Subject Classification. Primary 14H10, $14 \mathrm{H} 55$. 
1.2. Acknowledgments. We thank Eduard Looijenga for a continuous exchange of ideas on this topic and the referee for useful suggestions.

\section{Linear series of Weierstrass type}

Below we describe a slight variation of the theory of deformation of $g_{d}^{1}$ 's and $g_{d}^{2}$ 's on smooth curves. That theory is described in Chapter XXI of [1]. Set

$$
\mathcal{G}_{d, *}^{r}=\left\{[(C, p, Z)] \mid 1 \in Z \subset H^{0}(C, d p), \operatorname{dim}(Z)=r+1\right\}
$$

which naturally sits inside the coarse space associated to $\mathcal{G}_{d}^{r} \times{ }_{\mathcal{M}_{g}} \mathcal{M}_{g, 1}$.

Following the same arguments to prove the smoothness and to compute the dimension of $\mathcal{G}_{d}^{1}$ as in [1], Chapter XXI, Proposition 6.8, one proves that the variety $\mathcal{G}_{d, *}^{1}$ is smooth and of dimension equal to $2 g+d-3$.

2.1. Plane curves. Let us next recall the basic setting for the study of $\mathcal{G}_{d}^{2}$ as described in Sections 8 , 9 and 10, Chapter XXI of $\mathbf{1}$.

Let $C$ be a smooth genus $g$ curve and $\varphi: C \rightarrow \mathbb{P}^{2}$ a nonconstant morphism. The normal sheaf $N_{\varphi}$ to this morphism is defined by the exact sequence

$$
0 \rightarrow T_{C} \stackrel{d \varphi}{\rightarrow} \varphi^{*} T_{\mathbb{P}^{2}} \rightarrow N_{\varphi} \rightarrow 0 .
$$

Let

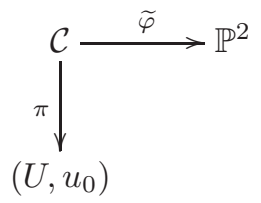

be a deformation of $\varphi$ parametrized by a pointed analytic space $\left(U, u_{0}\right)$, so that $\iota: C \stackrel{\cong}{\leftrightarrows} \pi^{-1}\left(u_{0}\right)$ and $\widetilde{\varphi} \circ \iota=\varphi$. The characteristic homomorphism of this family is the homomorphism

$$
T_{u_{0}}(U) \rightarrow H^{0}\left(C, N_{\varphi}\right)
$$

where $T_{u_{0}}(U)$ is the Zariski tangent space to $U$ at $u_{0}$, assigning to each tangent vector to $U$ at $u_{0}$ the Horikawa class of the corresponding infinitesimal deformation of $\varphi$. Denote by $N_{\varphi}^{\prime}$ the line bundle quotient of $N_{\varphi}$, i.e $N_{\varphi}^{\prime}=N_{\varphi} /$ Torsion. The line bundle $N_{\varphi}^{\prime}$ may also be defined by the exact sequence

$$
0 \rightarrow T_{C}(R) \stackrel{d \varphi}{\rightarrow} \varphi^{*} T_{\mathbb{P}^{2}} \rightarrow N_{\varphi}^{\prime} \rightarrow 0
$$

where $R$ be the ramification divisor of $\varphi$. In Proposition 9.10 (loc.cit) it is proved that, if the restriction of $\widetilde{\varphi}$ to each fiber of $\pi$ is birational, then for a general $u \in U$ the image of the characteristic homomorphism $T_{u}(U) \rightarrow H^{0}\left(C_{u}, N_{\varphi_{u}}\right)$ does not intersect the kernel of $H^{0}\left(C_{u}, N_{\varphi_{u}}\right) \rightarrow H^{0}\left(C_{u}, N_{\varphi_{u}}^{\prime}\right)$, where $C_{u}=\pi^{-1}(u)$ and $\varphi_{u}=\left.\widetilde{\varphi}\right|_{C_{u}}$ (here by general point of $U$ we mean a general point of one of its irreducible components).

A local universal deformation for a morphism $\varphi: C \rightarrow \mathbb{P}^{2}$ can be constructed as follows, at least when $g \geq 2$ (the cases $g=0,1$ are best treated separately). Let $d=\operatorname{deg} \varphi^{*}\left(\mathcal{O}_{\mathbb{P}^{2}}(1)\right)$, let $\mathcal{K} \rightarrow B$ be a Kuranishi family for $C$. Consider the Brill-Noether variety $\mathcal{G}_{d}^{2}$ over $B$ and let $\mathcal{V}$ be the bundle of projective frames for the universal $g_{d}^{2}$ over $\mathcal{G}_{d}^{2}$. Pulling back $\mathcal{K}$ to $\mathcal{V}$, yields a deformation of $\varphi$

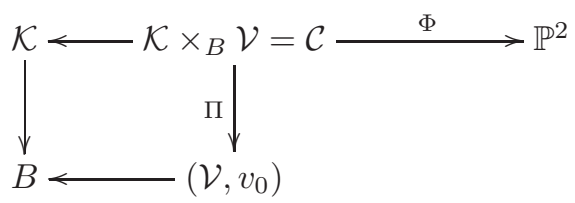

parametrized by $\mathcal{V}$, with $C \cong \Pi^{-1}\left(v_{0}\right)$ and $\varphi \cong \varphi_{v_{0}}=\left.\Phi\right|_{C_{v_{0}}}$. Moreover, for a general point $v \in \mathcal{V}$, the characteristic homomorphism yields an isomorphism

$$
T_{v}(\mathcal{V}) \cong H^{0}\left(C_{v}, N_{\varphi_{v}}\right) .
$$


In Theorem 10.1, Chapter XXI (loc.cit) it is proved that if $\mathcal{X}$ is an irreducible component of $\mathcal{G}_{d}^{2}$ whose general point corresponds to a curve $C$ of genus $g$ equipped with a basepoint-free $g_{d}^{2}$, which is not composed with an involution, then $\operatorname{dim} \mathcal{X}=3 d+g-9$, or equivalently $\operatorname{dim} \mathcal{V}^{\prime}=3 d+g-1$ where $\mathcal{V}^{\prime}:=\mathcal{V} \times \mathcal{G}_{d}^{2} \mathcal{X}$ is the pull-back to $\mathcal{X}$ of the bundle $\mathcal{V}$. There the theorem is proved under the assumption that $g \geq 2$, but the cases $g=0,1$ can be treated in a similar way.

In Theorem 10.14, Chapter XXI (loc.cit) it is proved that, for every (non-negative) value of $d$ and $g$ such that $(d-1)(d-2) / 2 \geq g$, there exists a genus $g$ curve $C$ equipped with a basepoint-free $g_{d}^{2}$ which realizes $C$ as a plane nodal curve of degree $d$.

2.2. Plane curves with a total tangency point. In order to study $\mathcal{G}_{d, *}^{2}$ we need to consider the appropriate deformation problem. Let us fix a point $Q$ and a line $L$ in $\mathbb{P}^{2}$. Look at the deformation (2.1) and suppose that

(a) $\pi: \mathcal{C} \rightarrow U$ is a family of pointed curves i.e. $\pi$ has a section $\sigma$.

(b) For each $u \in U, \varphi_{u}(\sigma(u))=Q$.

(c) For each $u \in U, \varphi_{u}$ is birational.

(d) The plane curve $\Gamma_{u}=\varphi_{u}\left(C_{u}\right)$ is unibranched at $Q$ with tangent line $L$ intersecting $\Gamma_{u}$ in $Q$ with multiplicity $d=\operatorname{deg} \Gamma_{u}$.

Imitating the arguments in (loc.cit) one sees that the characteristic homomorphism (2.2) factors through the inclusion $H^{0}\left(C, N_{\varphi}(-d p)\right) \subset H^{0}\left(C, N_{\varphi}\right)$, where $p=\sigma\left(u_{0}\right)$ and that, moreover, for a general $u \in U$ the image of the characteristic homomorphism does not intersect the kernel of $H^{0}\left(C, N_{\varphi}(-d p)\right) \rightarrow H^{0}\left(C, N_{\varphi}^{\prime}(-d p)\right)$.

Assume now $g \geq 2$; the cases $g=0,1$ can be easily dealt with separately.

Consider the natural morphism $\tau: \mathcal{G}_{d, *}^{2} \rightarrow \mathcal{G}_{d}^{2}$, which is finite-to-one, and its restriction to the irreducible component $\mathcal{X}$. An element of $\mathcal{V}^{\prime}$ corresponds to a triple $(C, p, \varphi)$, where $\varphi: C \rightarrow \mathbb{P}^{2}$ is the morphism associated to a frame of a subspace $Z \subset H^{0}(C, d p)$, with $[(C, p, Z)] \in \mathcal{X}$. Fix a point $Q$ and a line $L$ in $\mathbb{P}^{2}$. Let $\mathcal{V}^{*}$ be the subbundle of $\mathcal{V}^{\prime}$ given by those frames having the property that the corresponding morphism $\varphi: C \rightarrow \mathbb{P}^{2}$ is such that $\varphi(p)=Q$ and $\varphi^{*}(L)=d p$. Hence, if $\mathcal{C}^{*}$ is the restriction of the family $\mathcal{C} \rightarrow \mathcal{V}$ over $\mathcal{V}^{*}$, the family

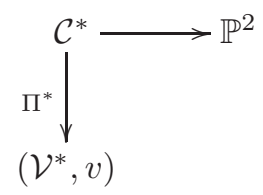

satisfies conditions (a), (b) and (d) above and it is a local universal deformation.

Theorem 10.1, Chapter XXI (loc.cit), in the present situation, translates into the following.

LEMma 2.1. Let $\mathcal{X}$ be an irreducible component of $\mathcal{G}_{d, *}^{2}$ whose general point corresponds to a triple $(C, p, Z)$, where $(C, p)$ is a genus $g$ pointed curve and $Z$ is a three-dimensional subspace of $H^{0}(C, d p)$, with $1 \in Z$ and whose corresponding $g_{d}^{2} \subset|d p|$ is basepoint-free and not composed with an involution. Then $\operatorname{dim} \mathcal{X}=2 d+g-6$.

Proof. Assume that $g \geq 2$. The cases $g=0,1$ can be easily treated separately. For our purposes we may restrict our attention to a small neighbourhood of $[(C, p, Z)]$ in $\mathcal{X}$. By assumption, $h^{0}(d p)=l>2$ and the linear series $|d p|$ is fixed point free and not composed with an involution; so, the family $\Pi^{*}$ satisfies also condition (c) above.

Clearly,

$$
\operatorname{dim} \mathcal{V}^{*}=\operatorname{dim} \mathcal{X}+5
$$

Since, for a plane curve, imposing a $d$-fold tangency with a given line $L$ at given point $Q$ amounts to $d$ linear conditions, we also have

$$
\operatorname{dim} \mathcal{V}^{*} \geq \operatorname{dim} \mathcal{V}^{\prime}-d=2 d+g-1
$$


If $v$ is a general point of $\mathcal{V}^{*}$ corresponding to a point $[(C, p, Z)] \in \mathcal{X}$ and a morphism $\varphi: C \rightarrow \mathbb{P}^{2}$, we get a commutative diagram

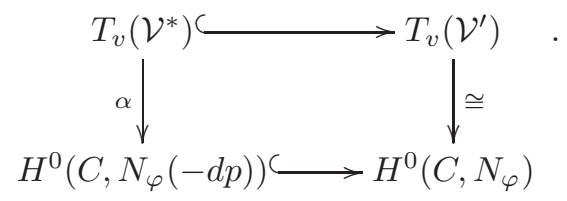

Since the image of $\alpha$ does not intersect the kernel of $H^{0}\left(C, N_{\varphi}(-d p)\right) \rightarrow H^{0}\left(C, N_{\varphi}^{\prime}(-d p)\right)$, we get

$$
\operatorname{dim} \mathcal{V}^{*} \leq h^{0}\left(N_{\varphi}^{\prime}(-d p)\right) .
$$

From (2.6) it follows that the line bundle $N_{\varphi}^{\prime}(-d p)$ is non-special so that $H^{1}\left(C, N_{\varphi}^{\prime}(-d p)\right)=H^{1}\left(C, N_{\varphi}(-d p)\right)=$ 0 . On the other hand, via the Euler sequence, we get

$$
N_{\varphi}^{\prime}=\omega_{C}(-R) \otimes \phi^{*} \mathcal{O}_{\mathbb{P}^{2}}(3)
$$

By Riemann-Roch, we get

$$
\begin{aligned}
\operatorname{dim} \mathcal{V}^{*} & \leq\left(\operatorname{deg}\left(N_{\varphi}^{\prime}\right)-d\right)-g+1=2 g-2-\operatorname{deg}(R)+3 d-d-g+1 \\
& =g+2 d-1-\operatorname{deg}(R) \leq g+2 d-1 .
\end{aligned}
$$

The lemma follows now from the above inequality, together with (2.5) and (2.6).

Lemma 2.2. For every $g$ and $d$ such that $(d-1)(d-2) \geq 2 g$ there exists a genus $g$ pointed curve $(C, p)$ equipped with a basepoint-free $g_{d}^{2} \subset|d p|$ which realizes $C$ as a plane nodal curve $\Gamma$ of degree $d$, with a smooth point (the image of $p$ ) whose tangent line has intersection multiplicity $d$ with $\Gamma$.

Proof. Here again to we use a slight variation of the arguments used in Section 10 of Chapter XXI (loc.cit). Fix a point $Q$ and a line $L$ in $\mathbb{P}^{2}$ and denote by $\Sigma_{d, g}^{*}$ the continuous system of all irreducible plane curves $\Gamma$ of degree $d$ whose normalization has genus $g$, and such that $(\Gamma \cdot L)_{Q}=d$. Let $\varphi: C \rightarrow \Gamma$ be the normalization. We consider an irreducible component $\Sigma^{*}$ of $\Sigma_{d, g}^{*}$ having the property that its general member represents a plane curve which is smooth at $Q$. Then one proves that (if non-empty) $\Sigma^{*}$ has dimension equal to $2 d+g-1$ and that a general point of $\Sigma^{*}$ corresponds to a plane irreducible curve of degree $d$ having $\delta=(d-1)(d-2) / 2-g$ nodes, and no other singularity. The proof of this fact is, word by word, the proof of Theorem 10.7 (loc.cit), where one should substitute the normal sheaf $N_{\varphi}$ with $N_{\varphi}(-d p)$. To prove that a non empty component $\Sigma^{*}$ exists, one may proceed as follows. Looking at the curve $y=x^{d}$ one sees that $\Sigma_{d, 0}^{*}$ is non empty. Let $\Gamma_{0}$ be a rational nodal curve corresponding to a general point of (a component) of $\Sigma_{d, 0}^{*}$. Mimicking the arguments used to prove Lemma 10.15 (loc.cit), but again using $N_{\varphi}(-d p)$ instead of $N_{\varphi}$, one shows that, given any integer $k$ with $0 \leq k \leq(d-1)(d-2) / 2$, there exists a deformation of $\Gamma_{0}$ whose general member is a plane irreducible curve $\Gamma$ having $\delta=(d-1)(d-2) / 2-k$ nodes and no other singularity, and having $Q$ as a simple point with $(\Gamma \cdot L)_{Q}=d$.

\section{On the Weierstrass flags}

Let $g$ and $d$ be integer such that $2 \leq d \leq g+1$. Consider the closed subvariety

$$
\bar{W}_{*}(d)=\left\{[(C, p)] \in M_{g, 1} \mid h^{0}(C, d p) \geq 2\right\}
$$

of $M_{g, 1}$ of dimension $2 g-3+d$ and notice that

$$
\bar{W}_{*}(2) \subset \bar{W}_{*}(3) \subset \cdots \subset \bar{W}_{*}(g) \subset \bar{W}_{*}(g+1)=M_{g, 1}
$$

is a stratification of $M_{g, 1}$, whose locally closed strata are given by

$$
W_{*}(d)=\bar{W}_{*}(d) \backslash \bar{W}_{*}(d-1)=\left\{[(C, p)] \in M_{g} \mid h^{0}(C, d p)=2\right\} .
$$

Similarly, consider the closed subvariety of $M_{g}$ of dimension $2 g-3+d$

$$
\bar{W}(d)=\pi\left(\bar{W}_{*}(d)\right)=\left\{[C] \in M_{g} \mid \exists p \in C \text { with } h^{0}(C, d p) \geq 2\right\}
$$

where $\pi: M_{g, 1} \rightarrow M_{g}$ is the forgetful morphism. Then

$$
\bar{W}(2) \subset \bar{W}(3) \subset \cdots \subset \bar{W}(g)=M_{g}
$$


is a stratification of $M_{g}$, whose locally closed strata are given by

$$
W(d)=\bar{W}(d) \backslash \bar{W}(d-1)=\left\{\begin{array}{l|l}
{[C] \in M_{g}} & \begin{array}{l}
\exists p \in C \text { with } h^{0}(C, d p)=2, \text { and } \\
h^{0}(C,(d-1) q)=1 \text { for all } q \in C
\end{array}
\end{array}\right\} .
$$

Consider first the strata $W_{*}(d)$ of $M_{g, 1}$.

Proposition 3.1. Let $5 \leq d \leq g+1$. If $d$ is not prime, or if $d$ is a prime and $(d-1)(d-2) \geq 2 g$, then $W_{*}(d)$ is not affine.

Proof. There is a natural forgetful morphism

$$
\begin{gathered}
\mathcal{G}_{d, *}^{1} \longrightarrow \bar{W}_{*}(d) \\
{[(C, p, Z)] \longmapsto[(C, p)]}
\end{gathered}
$$

that restricts to an isomorphism

$$
\mathcal{G}_{d, *}^{1} \backslash\left(\mathcal{G}_{d-1, *}^{1} \cup \mathcal{Z}\right) \cong W_{*}(d)
$$

where

$$
\mathcal{Z}=\left\{[(C, p, Z)] \in \mathcal{G}_{d, *}^{1} \mid 1 \in Z \subset H^{0}(C, d p), \operatorname{dim}(Z)=2, h^{0}(d p)>2\right\} .
$$

The variety $\mathcal{G}_{d, *}^{1}$ is smooth and of dimension equal to $2 g+d-3$. Since $\mathcal{G}_{d-1, *}^{1} \subset \mathcal{G}_{d, *}^{1}$ is a divisor, in order to show that $W_{*}(d)$ is not affine we will prove that

(I) $\mathcal{Z} \nsubseteq \mathcal{G}_{d-1, *}^{1}$

(II) every irreducible component $\mathcal{Z}^{\prime}$ of $\mathcal{Z}$ not entirely contained in $\mathcal{G}_{d-1, *}^{1}$ satisfies the condition: $\operatorname{codim}_{\mathcal{G}_{d, *}^{1}} \mathcal{Z}^{\prime}>1$.

First suppose that $d$ is not prime and set $d=h k$, with $h>1$ and $k>1$. Let $C$ be a genus $g$, $k$-sheeted cover of $\mathbb{P}^{1}$ with a point $p$ of total ramification. Since $d=h k$, with $h>1$, we may choose an element $f \in H^{0}(C, d p) \backslash H^{0}(C,(d-1) p)$ and set $Z=\langle 1, f\rangle$. Then $[(C, p, Z)] \in \mathcal{Z} \backslash \mathcal{Z} \cap \mathcal{G}_{d-1, *}^{1}$. This proves (I). We next turn our attention to point (II).

Let $\mathcal{Z}^{\prime}$ be an irreducible component of $\mathcal{Z}$ not entirely contained in $\mathcal{G}_{d-1, *}^{1}$. We can assume that a general point of $\mathcal{Z}^{\prime}$ corresponds to a triple $\left(C, p, Z\right.$ ), as above, with $h^{0}(d p)=l>2$ and $h^{0}((d-1) p)=l-1$ (no fixed points for $|d p|)$.

Two cases may occur:

(i) At a general point of $\mathcal{Z}^{\prime}$, the linear series $|d p|$ is composed with an involution.

(ii) At a general point of $\mathcal{Z}^{\prime}$, the linear series $|d p|$ is not composed with an involution.

Case (i).

The curve $C$ is a $\nu$-sheeted cover of curve $\Gamma$, with $d=k \nu$, and with a point of total ramification. Let $F: C \rightarrow \Gamma$ be this cover and let $\gamma$ be the genus of $\Gamma$. The number $\delta$ of branch points of $F$ (including $F(p)$ ) is at most

Therefore,

$$
\delta \leq 2 g-2 \nu(\gamma-1)-\nu .
$$

$$
\begin{aligned}
\operatorname{dim} \mathcal{Z}^{\prime} & \leq \delta+(2 \gamma+k-3) & & \\
& \leq 2 g+(2-2 \nu)(\gamma-1)-\nu-k-1, & & \text { if } \gamma \geq 1, \\
\operatorname{dim} \mathcal{Z}^{\prime} & \leq[(\delta-1)-2]+[(k+1)-2] \leq 2 g+\nu+k-4, & & \text { if } \quad \gamma=0 .
\end{aligned}
$$

In all cases, using the fact that $d=k \nu$ and $k, \nu>1$, one sees that

$$
\operatorname{dim} \mathcal{Z}^{\prime} \leq d+2 g-5=\operatorname{dim} \mathcal{G}_{d, *}^{1}-2 .
$$

is always satisfied except for $d<5, k=\nu=2$ and $\gamma=0$. Hence, the inequality always holds if $d \geq 5$.

\section{Case (ii).}


Let $[(C, p, Z)]$ be a general point of $\mathcal{Z}^{\prime}$. By assumption $h^{0}(d p)=l>2$ and the linear series $|d p|$ is fixed point free and not composed with an involution. There is a natural $\mathbb{P}^{l-3}$-bundle $\mathcal{P}$ over $\mathcal{Z}^{\prime}$ whose fiber over $[(C, p, Z)]$ is $\mathbb{P}\left(H^{0}(C, d p) / Z\right)$. A point of this bundle over $[(C, p, Z)]$ can be viewed as a triple $[(C, p, P)]$, where $P$ is a 3 -dimensional subspace of $H^{0}(C, d p)$ containing $Z$. Thus, there is an injective map

$$
\mathcal{P} \rightarrow \mathcal{G}_{d, *}^{2} .
$$

Therefore, by Lemma 2.1,

$$
\operatorname{dim} \mathcal{Z}^{\prime}=\operatorname{dim} \mathcal{P}-(l-3) \leq 2 d+g-3-l .
$$

As $l \geq 3$ and $d \leq g+1$, we obtain $2 d+g-3-l \leq 2 g+d-5$, and so the codimension of $\mathcal{Z}^{\prime}$ in $\mathcal{G}_{d, *}^{1}$ is strictly greater than 1 , proving the first part of the proposition.

Suppose next that $(d-1)(d-2) / 2-g \geq 0$. Then, by Lemma 2.2, there exists an irreducible component $\mathcal{Z}^{\prime}$ of $\mathcal{Z}$ which falls in case (ii) and again we are done.

Now we turn our attention to the locally closed strata $W(d)$ of $M_{g}$.

Proposition 3.2. Let $g \geq 6$ and $5 \leq d \leq g-1$. Then $W(d)$ is not affine.

PRoof. The forgerful map $\pi$ restricts to

$$
\left.\pi\right|_{\bar{W}_{*}(d)}: \bar{W}_{*}(d) \rightarrow \bar{W}(d)
$$

and, in particular, to

$$
\left.\pi\right|_{W_{*}(d) \backslash \mathcal{Y}}: W_{*}(d) \backslash \mathcal{Y} \rightarrow W(d)
$$

which is finite and surjective, where

$$
\mathcal{Y}=\left\{[(C, p)] \in W_{*}(d) \mid \exists q \neq p, \text { with } h^{0}(C,(d-1) q) \geq 2\right\} .
$$

Hence, $W(d)$ is affine only if $W_{*}(d) \backslash \mathcal{Y}$ is. Since $W_{*}(d)$ is smooth, to show that $W(d)$ is affine it suffices to find a non-empty component $\mathcal{Y}^{\prime}$ of $\mathcal{Y}$ of codimension greater than 1 inside $W_{*}(d)$. This is a direct consequence of Theorem 3.2 in Diaz's paper [6]. There the following is proven.

Theorem $3.3\left([\mathbf{6})\right.$. Let $g \geq 4$ and let $k \leq l \leq g-1$. If $k, l \geq \frac{1}{2}(g+2)$, then there exists a non-empty component $W(k, l)$ of the locus of points in $M_{g}$ corresponding to curves possessing both a Weierstrass point of type $k$ and a Weierstrass point of type $l$ which has dimension $g-3+k+l$.

A closer inspection of Diaz' proof also shows that:

For $g \geq 4$ and $k \leq l \leq g-1$, if $k<\frac{1}{2}(g+2)$, then there exists a non-empty component $W(k, l)$ of the locus of points in $M_{g}$ corresponding to curves possessing both a Weierstrass point of type $k$ and a Weierstrass point of type $l$ which has dimension at most $2 g-1+l-k$.

By a Weierstrass point of type $h$ on a curve $C$ it is a meant a point $p \in C$ for which $h^{0}(C, h p) \geq 2$. In proving this assertion, Diaz also shows that, if $k<l$, then for a general $[C] \in W(k, l)$ there exists a point $p \in C$ with $h^{0}(C, l p)=2$. We can then take

$$
\mathcal{Y}^{\prime}=\pi^{-1}(W(d-1, d)) \cap W_{*}(d) .
$$

Hence, for $4+g / 2 \leq d \leq g-1$,

$$
\operatorname{dim} \mathcal{Y}^{\prime}=g-3+(d-1)+d=g+2 d-4 \leq d+2 g-5=\operatorname{dim} W_{*}(d)-2
$$

On the other hand, for $5 \leq d<4+g / 2$,

$$
\operatorname{dim} \mathcal{Y}^{\prime} \leq 2 g-1+d-(d-1)=2 g \leq d+2 g-5=\operatorname{dim} W_{*}(d)-2 .
$$




\section{References}

1. E. Arbarello, M. Cornalba, and P. A. Griffiths, Geometry of algebraic curves. Vol. II with a contribution by $j$. d. harris, Grundlehren der Mathematischen Wissenschaften [Fundamental Principles of Mathematical Sciences], vol. 268, SpringerVerlag, New York, 2011. MR 770932 (86h:14019)

2. Enrico Arbarello, Weierstrass points and moduli of curves, Compositio Math. 29 (1974), 325-342. MR 0360601 (50 \#13048)

3. Enrico Arbarello and Maurizio Cornalba, On a conjecture of Petri, Comment. Math. Helv. 56 (1981), no. 1, 1-38. MR 615613 (82k:14029)

4. Lucia Caporaso and Joe Harris, Counting plane curves of any genus, Invent. Math. 131 (1998), no. 2, 345-392. MR 1608583 (99i:14064)

5. Steven Diaz, $A$ bound on the dimensions of complete subvarieties of $\mathcal{M}_{g}$, Duke Math. J. 51 (1984), no. 2, 405-408. MR $747872(85 \mathrm{j}: 14042)$

6. L Moduli of curves with two exceptional Weierstrass points, J. Differential Geom. 20 (1984), no. 2, 471-478. MR 788291 (86f:14012)

7. Claudio Fontanari and Eduard Looijenga, A perfect stratification of $\mathcal{M}_{g}$ for $g \leq 5$, Geom. Dedicata 136 (2008), $133-143$. MR 2443348 (2009f:14050)

8. Claudio Fontanari and Stefano Pascolutti, An affine open covering of $\mathcal{M}_{g}$ for $g \leq 5$, e-print arXiv:1103.2646, 2011.

9. Alexandre Grothendieck, Esquisse d'un programme, Geometric Galois actions, 1, London Math. Soc. Lecture Note Ser., vol. 242, Cambridge Univ. Press, Cambridge, 1997, With an English translation on pp. 243-283, pp. 5-48. MR 1483107 (99c:14034)

10. John L. Harer, The virtual cohomological dimension of the mapping class group of an orientable surface, Invent. Math. 84 (1986), no. 1, 157-176. MR 830043 (87c:32030)

11. Eduard Looijenga, On the tautological ring of $\mathcal{M}_{g}$, Invent. Math. 121 (1995), no. 2, 411-419. MR 1346214 (96g:14021)

12. David Mumford, Towards an enumerative geometry of the moduli space of curves, Arithmetic and geometry, Vol. II, Progr. Math., vol. 36, Birkhäuser Boston, Boston, MA, 1983, pp. 271-328. MR 717614 (85j:14046)

13. H. E. Rauch, Weierstrass points, branch points, and moduli of Riemann surfaces, Comm. Pure Appl. Math. 12 (1959), 543-560. MR 0110798 (22 \#1666)

Università di Roma "La Sapienza" - Dipartimento di Matematica - Piazzale Aldo Moro 5, 00185 Roma - Italy

E-mail address: ea@mat.uniroma1.it

Università di Roma "La Sapienza" - Dipartimento di Matematica - Piazzale Aldo Moro 5, 00185 Roma - Italy

E-mail address: mondello@mat.uniroma1.it 